\title{
Article \\ Multiple Comparison Procedures for Exponential Mean Lifetimes Compared with Several Controls
}

\author{
Shu-Fei Wu
}

check for updates

Citation: Wu, S.-F. Multiple Comparison Procedures for Exponential Mean Lifetimes Compared with Several Controls. Mathematics 2022, 10, 609. https:// doi.org/10.3390/math10040609

Academic Editors: Francisco Ureña and Leonid Piterbarg

Received: 23 December 2021 Accepted: 15 February 2022 Published: 16 February 2022

Publisher's Note: MDPI stays neutral with regard to jurisdictional claims in published maps and institutional affiliations.

Copyright: (C) 2022 by the author. Licensee MDPI, Basel, Switzerland. This article is an open access article distributed under the terms and conditions of the Creative Commons Attribution (CC BY) license (https:// creativecommons.org/licenses/by/ $4.0 /)$.
Department of Statistics, Tamkang University, Taipei 251301, Taiwan; 100665@mail.tku.edu.tw

\begin{abstract}
Under heteroscedasticity, we propose one-stage multiple comparison procedures for several treatment groups compared with several control groups in terms of exponential mean lifetimes. The simultaneous confidence intervals including one-sided and two-sided confidence intervals for the difference between the mean lifetime from the $i$-th treatment group and the mean lifetime from the $j$-th control group are developed in this research. The required critical values are obtained and tabulated for the practical use of users. The experimenters can use these simultaneous confidence intervals to determine whether the treatment mean lifetimes are better than several controls or worse than several controls under a specified confidence level. Finally, one example of comparing the mean duration of remission using four drugs for treating leukemia is used for the aims of illustrations.
\end{abstract}

Keywords: one-stage procedure; multiple comparison with the control; multiple comparison with several controls; exponential distribution

\section{Introduction}

Our research is related to the field of ranking and selection. For normal distributions, Bechhofer [1] and Gupta [2] are the pioneers in this field. Instead of normal distributions, exponential distributions are widely used to model the lifetimes of products. For exponential lifetime distributions, $k(\geq 2)$ independent treatment groups and $l$ control groups are considered. Let $X_{i 1}, \cdots, X_{i m}$ be the random sample of size $m$ from the $i$-th treatment group $\pi_{i}, I=1, \ldots, k$, where $\pi_{i}$ follows a two-parameter exponential distribution denoted by $E\left(\theta_{i}, \sigma_{i}\right), I=1, \ldots, k$. The parameters $\theta_{1}, \cdots, \theta_{k}$ are the unknown location parameters (also known as the guaranteed time in reliability and engineering) and the parameters $\sigma_{1}, \cdots, \sigma_{k}$ are the unknown and unequal scale parameters. Likewise, let $X_{j 1}^{*}, \cdots, X_{j m}^{*}$ be the random sample of size $m$ from the $j$-th control group $\pi_{j}^{*}, j=1, \ldots, l$, where $\pi_{j}^{*}$ follows a two-parameter exponential distribution denoted by $E\left(\eta_{j}, \xi_{j}\right), j=1, \ldots, l$. The parameters $\eta_{1}, \cdots, \eta_{l}$ are the unknown location parameters and parameters $\xi_{1}, \cdots, \xi_{l}$ are the unknown and unequal scale parameters. Furthermore, the mean lifetime for the $i$-th treatment group is $\mu_{i}=\theta_{i}+\sigma_{i}, I=1, \ldots, k$ and the mean lifetime for the $j$-th control group is $\mu_{j}^{*}=\eta_{j}+\xi_{j}$, $j=1, \ldots, l$. When scale parameters for exponential distributions are unknown and possibly unequal, Lam and $\mathrm{Ng}$ [3] proposed the design-oriented two-stage multiple comparisons with the control. Please see Lam and $\mathrm{Ng}$ [3] for the detailed introduction for the two-stage procedures. The disadvantage for the two-stage procedure is that the required sample size for the second stage may be large. To remedy this problem, the one-stage sampling procedure addressed above can be considered instead. Based on the one-stage sample from $\mathrm{k}$ populations, Wu et al. [4] developed the multiple comparison procedures for exponential location parameters with the control when scale parameters are unknown and unequal. Instead of comparing with the control, $\mathrm{Wu}$ [5] proposed one-stage multiple comparisons with the average for exponential location parameters. Maurya [6] investigated the one-stage multiple comparisons with more than one control under heteroscedasticity. Maurya [7] proposed another one-stage multiple comparisons with a control under heteroscedasticity. Instead of comparing the location parameters for exponential distributions, the one-stage 
multiple comparisons with the average in terms of mean lifetimes are proposed by $\mathrm{Wu}$ [8]. Unlike the multiple comparison procedure with the control based on mean lifetimes in $\mathrm{Wu}$ [8], the aim and the novelty of this paper is to compare several treatment populations with more than one control populations based on the mean lifetimes. The research methodologies are addressed in Section 2 by using the techniques in Lam's research $([9,10])$. The research results in $\mathrm{Wu}$ [8] can be treated as a special case of our proposed method in this paper when the number of control populations $l=1$. Therefore, this paper has more general applications compared with the results in $\mathrm{Wu}$ [8]. In order to illustrate our proposed simultaneous confidence intervals in Section 2, we use an example of comparing two treatment drugs with two control drugs for treating leukemia in Section 3. In this example, each drug group consists of 20 patients and the dataset for each group passed the fitness test for exponential distribution by the use of the Gini test (Gail and Gastwirth [11]). In Lawless [12], they conducted a likelihood ratio asymptotic chi-squared test for the homogeneity of scale parameters and the results revealed that the scale parameters for four exponential distributions are significantly different. In Section 4, the conclusions related to our research methods are made.

\section{Comparing with Several Controls for Exponential Mean Lifetimes}

For the one-stage sample $X_{i 1}, \cdots, X_{i m}$ of size $m$ from the $i$-th treatment group, we let $Y_{i}=\min \left(X_{i 1}, \cdots, X_{i m}\right)$ and $S_{i}=\sum_{j=1}^{m}\left(X_{i j}-Y_{i}\right) /(m-1), I=1, \ldots, k$. Likewise, for the one-stage sample $X_{j 1}^{*}, \cdots, X_{j m}^{*}$ of size $m$ from the $j$-th control group, we let $Y_{j}^{*}=\min \left(X_{j 1}^{*}\right.$, $\left.\cdots, X_{j m}^{*}\right)$ and $S_{j}^{*}=\sum_{t=1}^{m}\left(X_{j t}^{*}-Y_{j}^{*}\right) /(m-1), j=1, \ldots, l$.

Before we start to construct the simultaneous confidence interval (SCI) for $\mu_{i}-\mu_{j}^{*}$, $I=1, \ldots, k, j=1, \ldots, l$, we need to consider the following random variables:

$$
\begin{gathered}
G_{i}=\left(-m S_{i} / \sigma_{i}+m-m\left(Y_{i}-\theta_{i}\right) / \sigma_{i}\right) /\left(S_{i} / \sigma_{i}\right), I=1, \ldots, k \text { and } \\
G_{j}^{*}=\left(-m S_{j}^{*} / \xi_{j}+m-m\left(Y_{j}^{*}-\eta_{j}\right) / \xi_{j}\right) /\left(S_{j}^{*} / \xi_{j}\right), j=1, \ldots, l .
\end{gathered}
$$

From Roussas [13], they indicated that $Q_{i}=2(m-1) S_{i} / \sigma_{i} \sim \chi_{2 m-2}^{2}$ and $Q_{j}^{*}=2(m-1)$ $S_{j}^{*} / \xi \sim \chi_{2 m-2}^{2}$, where $\chi_{2 m-2}^{2}$ represents a chi-squared distribution with $2 m-2$ degrees of freedom (df). Furthermore, $E_{i}=m\left(Y_{i}-\theta_{i}\right) / \sigma_{i} \sim \operatorname{Exp}(1)$ and $E_{j}^{*}=m\left(Y_{j}^{*}-\eta_{j}\right) / \xi_{j} \sim \operatorname{Exp}(1)$, where $\operatorname{Exp}(1)$ represents a standard exponential distribution. In addition, the random variables $E_{i}, Q_{i}, i=1, \ldots, k$ and $E_{j}^{*}, Q_{j}^{*}, j=1, \ldots, l$ are stochastically independent.

In terms of $E_{i}, Q_{i}, i=1, \ldots, k$ and $E_{j}^{*}, Q_{j}^{*}, j=1, \ldots, l$, the random variables $G_{i}, I=1$, $\ldots, k$ and $G_{j}^{*}, j=1, \ldots, l$ can be rewritten as follows:

$$
\begin{gathered}
G_{i}=\frac{-m S_{i} / \sigma_{i}+m-m\left(Y_{i}-\theta_{i}\right) / \sigma_{i}}{S_{i} / \sigma_{i}}=\frac{-2 m(m-1) S_{i} / \sigma_{i}+2(m-1)\left(m-m\left(Y_{i}-\theta_{i}\right) / \sigma_{i}\right)}{2(m-1) S_{i} / \sigma_{i}} \\
=-m+\frac{2(m-1)\left(m-E_{i}\right)}{Q_{i}}
\end{gathered}
$$

and

$$
G_{j}^{*}=\frac{-m S_{j}^{*} / \xi_{j}+m-m\left(Y_{j}^{*}-\eta_{j}\right) / \xi_{j}}{S_{j}^{*} / \xi_{j}}=-m+\frac{2(m-1)\left(m-E_{j}^{*}\right)}{Q_{j}^{*}} .
$$

Once the independent random variables $E_{i} \sim \operatorname{Exp}(1)$ and $Q_{i} \sim \chi_{2 m-2}^{2}$ are generated, the random variable $G_{i}$ can be thus generated, $I=1, \ldots, k$. Likewise, once the independent random variables $E_{i} \sim \operatorname{Exp}(1)$ and $Q_{i} \sim \chi_{2 m-2}^{2}$, are generated, the random variable $G_{j}^{*}$ can be generated, $j=1, \ldots, l$.

Now we can propose the one-stage multiple comparison procedures for exponential mean lifetimes with several controls denoted by $\mu_{i}-\mu_{j}^{*}, I=1, \ldots, k, j=1, \ldots, l$ as follows: 
Theorem 1. For a given $0<P<1$, let $\widetilde{c}=\underset{i=1, \cdots, k, j=1, \ldots, l}{\max }\left(\frac{S_{i}}{m}, \frac{S_{j}^{*}}{m}\right)$,

$$
\begin{aligned}
G_{i} & =\left(-m S_{i} / \sigma_{i}+m-m\left(Y_{i}-\theta_{i}\right) / \sigma_{i}\right) /\left(S_{i} / \sigma_{i}\right), I=1, \ldots, k \text { and } \\
G_{j}^{*} & =\left(-m S_{j}^{*} / \xi_{j}+m-m\left(Y_{j}^{*}-\eta_{j}\right) / \xi_{j}\right) /\left(S_{j}^{*} / \xi_{j}\right), j=1, \ldots, l .
\end{aligned}
$$

We have the following results:

(a) If $\widetilde{s}_{U}$ is the 100P-th percentile of the distribution of $\max \left(-G_{j}^{*}, G_{i}, G_{i}-G_{j}^{*}, i=1, \cdots, k\right.$, $j=1, \ldots, l)$, then we have

$$
\mathrm{P}\left(\mu_{i}-\mu_{j}^{*} \leq Y_{i}+S_{i}-Y_{j}^{*}-S_{j}^{*}+\widetilde{c s}_{U}, i=1, \cdots, k, j=1, \ldots, l\right) \geq P .
$$

Thus, $\left(-\infty, Y_{i}+S_{i}-Y_{j}^{*}-S_{j}^{*}+\widetilde{c S}_{U}\right)$ is a set of upper confidence intervals for $\mu_{i}-\mu_{j}^{*}$ with confidence coefficient $P, I=1, \ldots, k, j=1, \ldots, l$.

(b) If $\widetilde{s}_{L}$ is the 100 P-th percentile of the distribution of $\max \left(-G_{i}, G_{j}^{*}, G_{j}^{*}-G_{i}, i=1, \cdots, k, j=1\right.$, $\ldots, l)$, then we have

$$
\mathrm{P}\left(\mu_{i}-\mu_{j}^{*} \geq Y_{i}+S_{i}-Y_{j}^{*}-S_{j}^{*}-\widetilde{c S}_{L^{\prime}} i=1, \cdots, k, j=1, \ldots, l\right) \geq P .
$$

Thus, $\left(Y_{i}+S_{i}-Y_{j}^{*}-S_{j}^{*}-\widetilde{c s}_{L}, \infty\right)$ is a set of lower confidence intervals for $\mu_{i}-\mu_{j}^{*}$ with confidence coefficient $P, I=1, \ldots, k, j=1, \ldots, l$.

(c) If $\widetilde{s}_{t}$ is the $100 P$-th percentile of the distribution of $\max \left(\left|G_{i}\right|,\left|G_{j}^{*}\right|,\left|G_{j}^{*}-G_{i}\right|, i=1, \cdots, k\right.$, $j=1, \ldots, l)$, then we have

$$
\begin{gathered}
\mathrm{P}\left(Y_{i}+S_{i}-Y_{j}^{*}-S_{j}^{*}-\widetilde{c s}_{t} \leq \mu_{i}-\mu_{j}^{*} \leq Y_{i}+S_{i}-Y_{j}^{*}-S_{j}^{*}+\widetilde{c s}_{t}, i=1, \cdots, k,\right. \\
j=1, \ldots, l) \geq P
\end{gathered}
$$

Thus, $\left(Y_{i}+S_{i}-Y_{j}^{*}-S_{j}^{*} \pm \widetilde{c s_{t}}\right)$ is a set of simultaneous two-sided confidence intervals for $\mu_{i}-\mu_{j}^{*}$ with confidence coefficient $P, I=1, \ldots, k, j=1, \ldots, l$.

The technique given in Lam $([9,10])$ is described in the following lemma:

Lemma 1. If $X$ and $Y$ are two random variables, $a$ and $b$ are two positive constants, then $[a X \geq$ $b Y-d \max (a, b)] \supseteq[X \geq-d, Y \leq d$ and $X \geq Y-d]$.

The proof of Lemma 1 is given in Lam [1] and it is briefly given in the section of Appendix A. Using the technique in Lemma 1, the proof of Theorem 1 is also given in Appendix A.

Since the critical values $\widetilde{s}_{U}, \widetilde{s}_{L}$ and $\widetilde{s}_{t}$ in Theorem 1 are difficult to obtain, the Monte Carlo simulation method is used to find the critical values and the algorithm to obtain these critical values is given as follows:

Step 1: Generate $k$ independent random variables $E_{i} \sim \operatorname{Exp}(1)$ and $l$ independent random variables $E_{j}^{*} \sim \operatorname{Exp}(1)$. Generate another $k$ independent random variables $Q_{i} \sim \chi_{2(m-1)}^{2}$ and $l$ independent random variables $Q_{j}^{*} \sim \chi_{2(m-1)}^{2}$. Then, the $k+l$ independent random variables:

$$
\begin{gathered}
G_{i}=-m+2(m-1)\left(m-E_{i}\right) / Q_{i}=1, \ldots, \text { kand } \\
G_{j}^{*}=-m+2(m-1)\left(m-E_{j}^{*}\right) / Q_{j}^{*},=1, \ldots, l \text { can be generated. }
\end{gathered}
$$

Step 2: The values of $\max \left(-G_{j}^{*}, G_{i}, G_{i}-G_{j}^{*}, i=1, \cdots, k, j=1, \ldots, l\right)$,

$$
\max \left(-G_{i}, G_{j}^{*}, G_{j}^{*}-G_{i,} i=1, \cdots, k, j=1, \ldots, l\right) \text { and }
$$




$$
\max \left(\left|G_{i}\right|,\left|G_{j}^{*}\right|,\left|G_{j}^{*}-G_{i}\right|, i=1, \cdots, k, j=1, \ldots, l\right) \text { can be computed. }
$$

Step 3: Repeat Step 1 and Step 2 100,000 times. Then, the critical values $\widetilde{s}_{U}, \widetilde{s}_{L}$ and $\widetilde{s}_{t}$ can be obtained as the $100 P$-th empirical percentiles of

$$
\begin{gathered}
\max \left(-G_{j}^{*}, G_{i}, G_{i}-G_{j}^{*}, i=1, \cdots, k, j=1, \ldots, l\right), \\
\max \left(-G_{i}, G_{j}^{*}, G_{j}^{*}-G_{i,}, i=1, \cdots, k, j=1, \ldots, l\right) \text { and } \\
\max \left(\left|G_{i}\right|,\left|G_{j}^{*}\right|,\left|G_{j}^{*}-G_{i}\right|, i=1, \cdots, k, j=1, \ldots, l\right) \text { respectively. }
\end{gathered}
$$

The approximate critical values $\widetilde{s}_{U}, \widetilde{s}_{L}$, and $\widetilde{s}_{t}$ under $(k, l)=(2,2),(2,3),(2,4),(3,2),(3,3)$, $(4,2), m=2(1) 10(5) 30$ and $P=0.90,0.95$, and 0.975 are listed in Table 1 . From Table 1 , it can be seen that the approximate critical values $\widetilde{s}_{U}, \widetilde{s}_{L}$, and $\widetilde{s}_{t}$ are increasing while $k+l$ is increasing for any given $P$ and $m$ or while $P$ is increasing for any given $k, l$, and $m$. Let $L_{1}$ be the length of the two-sided confidence intervals for $\mu_{i}-\mu_{j}^{*}, I=1, \ldots, k, j=1, \ldots, l$ for the one-stage procedure, then we have $L_{1}=2 \widetilde{c s}_{t}$. From this equation, we can see that the larger the $k+l$, the larger the value of $\widetilde{s}_{t}$ and then the larger confidence length of $L_{1}$ for any given

\begin{tabular}{|c|c|c|c|c|c|c|c|c|c|c|c|}
\hline \multirow{2}{*}{$k$} & \multirow{2}{*}{$l$} & \multirow[b]{2}{*}{$m$} & \multicolumn{3}{|c|}{$P=0.90$} & \multicolumn{3}{|c|}{$P=0.95$} & \multicolumn{3}{|c|}{$P=0.975$} \\
\hline & & & $\tilde{s}_{U}$ & $\tilde{s}_{L}$ & $\tilde{s}_{t}$ & $\tilde{s}_{U}$ & $\tilde{s}_{L}$ & $\tilde{s}_{t}$ & $\tilde{s}_{U}$ & $\tilde{s}_{L}$ & $\tilde{s}_{t}$ \\
\hline \multirow[t]{13}{*}{2} & 2 & 2 & 24.32 & 24.36 & 49.17 & 50.12 & 50.52 & 100.37 & 100.68 & 102.56 & 202.85 \\
\hline & & 3 & 11.13 & 11.13 & 16.84 & 17.06 & 17.06 & 25.14 & 25.35 & 25.26 & 36.73 \\
\hline & & 4 & 9.51 & 9.52 & 13.23 & 13.34 & 13.38 & 18.07 & 18.10 & 18.19 & 24.08 \\
\hline & & 5 & 9.18 & 9.20 & 12.27 & 12.33 & 12.39 & 16.01 & 16.03 & 16.10 & 20.43 \\
\hline & & 6 & 9.22 & 9.22 & 12.02 & 12.11 & 12.11 & 15.31 & 15.35 & 15.36 & 19.02 \\
\hline & & 7 & 9.40 & 9.42 & 12.06 & 12.14 & 12.13 & 15.04 & 15.08 & 15.09 & 18.33 \\
\hline & & 8 & 9.65 & 9.64 & 12.22 & 12.30 & 12.28 & 15.07 & 15.12 & 15.10 & 18.16 \\
\hline & & 9 & 9.90 & 9.93 & 12.44 & 12.48 & 12.53 & 15.19 & 15.22 & 15.23 & 18.09 \\
\hline & & 10 & 10.21 & 10.23 & 12.72 & 12.78 & 12.79 & 15.39 & 15.42 & 15.43 & 18.24 \\
\hline & & 15 & 11.67 & 11.68 & 14.23 & 14.29 & 14.28 & 16.85 & 16.87 & 16.87 & 19.47 \\
\hline & & 20 & 13.02 & 13.03 & 15.70 & 15.75 & 15.75 & 18.35 & 18.36 & 18.39 & 20.98 \\
\hline & & 25 & 14.28 & 14.30 & 17.10 & 17.13 & 17.17 & 19.84 & 19.84 & 19.88 & 22.46 \\
\hline & & 30 & 15.48 & 15.42 & 18.41 & 18.49 & 18.44 & 21.27 & 21.33 & 21.26 & 24.01 \\
\hline \multirow[t]{13}{*}{2} & 3 & 2 & 26.30 & 35.08 & 61.24 & 53.65 & 71.91 & 124.81 & 107.23 & 145.94 & 252.28 \\
\hline & & 3 & 11.65 & 14.09 & 19.30 & 17.70 & 21.30 & 28.67 & 26.07 & 31.42 & 41.94 \\
\hline & & 4 & 9.96 & 11.57 & 14.83 & 13.84 & 16.01 & 20.08 & 18.67 & 21.54 & 26.65 \\
\hline & & 5 & 9.61 & 10.90 & 13.55 & 12.82 & 14.44 & 17.52 & 16.54 & 18.52 & 22.18 \\
\hline & & 6 & 9.68 & 10.81 & 13.20 & 12.57 & 13.95 & 16.61 & 15.81 & 17.42 & 20.47 \\
\hline & & 7 & 9.90 & 10.89 & 13.15 & 12.63 & 13.78 & 16.27 & 15.62 & 16.92 & 19.65 \\
\hline & & 8 & 10.14 & 11.09 & 13.28 & 12.78 & 13.87 & 16.18 & 15.58 & 16.80 & 19.33 \\
\hline & & 9 & 10.43 & 11.34 & 13.48 & 13.01 & 14.03 & 16.29 & 15.75 & 16.85 & 19.31 \\
\hline & & 10 & 10.73 & 11.65 & 13.75 & 13.29 & 14.29 & 16.48 & 15.94 & 17.01 & 19.38 \\
\hline & & 15 & 12.31 & 13.10 & 15.28 & 14.91 & 15.75 & 17.91 & 17.49 & 18.36 & 20.55 \\
\hline & & 20 & 13.76 & 14.51 & 16.80 & 16.47 & 17.21 & 19.46 & 19.09 & 19.85 & 22.05 \\
\hline & & 25 & 15.13 & 15.86 & 18.29 & 17.95 & 18.71 & 21.01 & 20.64 & 21.40 & 23.68 \\
\hline & & 30 & 16.39 & 17.10 & 19.67 & 19.38 & 20.05 & 22.54 & 22.23 & 22.88 & 25.34 \\
\hline \multirow[t]{5}{*}{2} & 4 & 2 & 28.14 & 46.00 & 73.85 & 56.73 & 94.80 & 150.80 & 114.03 & 190.63 & 303.81 \\
\hline & & 3 & 12.08 & 16.69 & 21.52 & 18.19 & 25.01 & 31.74 & 26.67 & 36.62 & 46.28 \\
\hline & & 4 & 10.25 & 13.17 & 16.07 & 14.15 & 18.05 & 21.66 & 18.96 & 24.10 & 28.58 \\
\hline & & 5 & 9.90 & 12.25 & 14.59 & 13.12 & 16.04 & 18.75 & 16.84 & 20.46 & 23.57 \\
\hline & & 6 & 9.98 & 11.99 & 14.08 & 12.86 & 15.27 & 17.60 & 16.08 & 18.97 & 21.59 \\
\hline
\end{tabular}
$P$ and $m$. Likewise, we can also see that when $P$ increases, the confidence length increases for any given $k, l$, and $m$.

Table 1. Critical values of $\widetilde{s}_{U}, \widetilde{s}_{L}$, and $\widetilde{s}_{t}$ for $P=0.90,0.95$, and 0.975 . 
Table 1. Cont.

\begin{tabular}{|c|c|c|c|c|c|c|c|c|c|c|c|}
\hline \multirow{2}{*}{$k$} & \multirow{2}{*}{$l$} & \multirow{2}{*}{$m$} & \multicolumn{3}{|c|}{$P=0.90$} & \multicolumn{3}{|c|}{$P=0.95$} & \multicolumn{3}{|c|}{$P=0.975$} \\
\hline & & & $\tilde{s}_{U}$ & $\tilde{s}_{L}$ & $\tilde{s}_{t}$ & $\tilde{s}_{U}$ & $\tilde{s}_{L}$ & $\tilde{s}_{t}$ & $\tilde{s}_{U}$ & $\tilde{s}_{L}$ & $\tilde{s}_{t}$ \\
\hline \multirow{21}{*}{3} & \multirow{21}{*}{2} & 7 & 10.19 & 11.99 & 13.97 & 12.92 & 15.01 & 17.15 & 15.88 & 18.29 & 20.70 \\
\hline & & 8 & 10.46 & 12.16 & 14.07 & 13.08 & 15.04 & 17.09 & 15.90 & 18.13 & 20.32 \\
\hline & & 9 & 10.76 & 12.41 & 14.28 & 13.36 & 15.18 & 17.15 & 16.05 & 18.12 & 20.18 \\
\hline & & 10 & 11.10 & 12.65 & 14.52 & 13.67 & 15.36 & 17.31 & 16.35 & 18.17 & 20.24 \\
\hline & & 15 & 12.76 & 14.13 & 16.05 & 15.33 & 16.78 & 18.70 & 17.88 & 19.42 & 21.38 \\
\hline & & 20 & 14.32 & 15.59 & 17.63 & 17.01 & 18.28 & 20.29 & 19.63 & 20.90 & 22.92 \\
\hline & & 25 & 15.70 & 16.96 & 19.13 & 18.51 & 19.76 & 21.87 & 21.22 & 22.45 & 24.51 \\
\hline & & 30 & 17.04 & 18.25 & 20.57 & 20.01 & 21.19 & 23.41 & 22.81 & 24.00 & 26.16 \\
\hline & & 2 & 35.20 & 26.44 & 61.78 & 72.37 & 54.14 & 126.61 & 147.01 & 108.88 & 255.82 \\
\hline & & 3 & 14.11 & 11.73 & 19.34 & 21.36 & 17.76 & 28.75 & 31.50 & 26.19 & 41.94 \\
\hline & & 4 & 11.54 & 9.95 & 14.79 & 15.96 & 13.82 & 20.01 & 21.39 & 18.67 & 26.57 \\
\hline & & 5 & 10.90 & 9.62 & 13.56 & 14.44 & 12.82 & 17.54 & 18.57 & 16.52 & 22.21 \\
\hline & & 6 & 10.81 & 9.69 & 13.21 & 13.95 & 12.58 & 16.65 & 17.48 & 15.83 & 20.50 \\
\hline & & 7 & 10.88 & 9.87 & 13.15 & 13.79 & 12.61 & 16.25 & 16.93 & 15.60 & 19.68 \\
\hline & & 8 & 11.07 & 10.12 & 13.25 & 13.84 & 12.75 & 16.17 & 16.78 & 15.57 & 19.28 \\
\hline & & 9 & 11.36 & 10.43 & 13.50 & 14.09 & 13.02 & 16.32 & 16.93 & 15.71 & 19.29 \\
\hline & & 10 & 11.62 & 10.76 & 13.75 & 14.27 & 13.32 & 16.50 & 17.03 & 15.98 & 19.37 \\
\hline & & 15 & 13.09 & 12.30 & 15.25 & 15.71 & 14.87 & 17.89 & 18.35 & 17.46 & 20.53 \\
\hline & & 20 & 14.52 & 13.78 & 16.82 & 17.25 & 16.48 & 19.50 & 19.91 & 19.13 & 22.13 \\
\hline & & 25 & 15.85 & 15.14 & 18.28 & 18.67 & 17.98 & 21.02 & 21.37 & 20.70 & 23.69 \\
\hline & & 30 & 17.08 & 16.40 & 19.66 & 20.04 & 19.38 & 22.51 & 22.84 & 22.18 & 25.23 \\
\hline \multirow[t]{13}{*}{3} & \multirow[t]{13}{*}{3} & 2 & 36.89 & 37.08 & 71.42 & 75.84 & 76.37 & 149.80 & 154.64 & 151.29 & 307.21 \\
\hline & & 3 & 14.75 & 14.65 & 20.62 & 21.62 & 21.84 & 30.33 & 32.03 & 32.58 & 45.30 \\
\hline & & 4 & 11.93 & 11.96 & 15.14 & 16.50 & 16.32 & 20.75 & 21.69 & 22.07 & 27.54 \\
\hline & & 5 & 11.34 & 11.33 & 13.72 & 14.91 & 14.92 & 17.98 & 18.98 & 19.23 & 22.82 \\
\hline & & 6 & 11.18 & 11.21 & 13.19 & 14.41 & 14.38 & 16.75 & 17.81 & 17.80 & 20.63 \\
\hline & & 7 & 11.35 & 11.39 & 13.09 & 14.23 & 14.33 & 16.23 & 17.41 & 17.31 & 19.67 \\
\hline & & 8 & 11.60 & 11.59 & 13.13 & 14.26 & 14.34 & 16.06 & 17.34 & 17.34 & 19.34 \\
\hline & & 9 & 11.89 & 11.84 & 13.27 & 14.63 & 14.63 & 16.24 & 17.43 & 17.37 & 19.13 \\
\hline & & 10 & 12.17 & 12.18 & 13.48 & 14.78 & 14.82 & 16.21 & 17.64 & 17.51 & 19.15 \\
\hline & & 15 & 13.70 & 13.81 & 14.79 & 16.35 & 16.33 & 17.44 & 19.08 & 18.86 & 20.20 \\
\hline & & 20 & 15.23 & 15.33 & 16.20 & 17.95 & 17.98 & 18.87 & 20.59 & 20.67 & 21.55 \\
\hline & & 25 & 16.74 & 16.71 & 17.60 & 19.39 & 19.54 & 20.19 & 22.18 & 22.23 & 23.09 \\
\hline & & 30 & 18.06 & 18.07 & 18.86 & 20.93 & 20.89 & 21.70 & 23.63 & 23.77 & 24.37 \\
\hline \multirow[t]{13}{*}{4} & \multirow[t]{13}{*}{2} & 2 & 46.06 & 28.21 & 73.93 & 94.26 & 57.19 & 150.66 & 189.64 & 114.78 & 301.99 \\
\hline & & 3 & 16.63 & 12.08 & 21.46 & 24.99 & 18.16 & 31.72 & 36.63 & 26.62 & 46.31 \\
\hline & & 4 & 13.19 & 10.23 & 16.07 & 18.01 & 14.11 & 21.60 & 24.00 & 18.93 & 28.49 \\
\hline & & 5 & 12.26 & 9.90 & 14.59 & 16.05 & 13.08 & 18.77 & 20.50 & 16.83 & 23.66 \\
\hline & & 6 & 11.98 & 9.95 & 14.09 & 15.30 & 12.86 & 17.64 & 18.99 & 16.10 & 21.62 \\
\hline & & 7 & 12.02 & 10.19 & 14.01 & 15.06 & 12.92 & 17.21 & 18.36 & 15.92 & 20.74 \\
\hline & & 8 & 12.17 & 10.47 & 14.09 & 15.05 & 13.12 & 17.10 & 18.13 & 15.95 & 20.35 \\
\hline & & 9 & 12.37 & 10.78 & 14.25 & 15.14 & 13.38 & 17.13 & 18.06 & 16.11 & 20.19 \\
\hline & & 10 & 12.66 & 11.12 & 14.54 & 15.39 & 13.68 & 17.32 & 18.20 & 16.35 & 20.24 \\
\hline & & 15 & 14.12 & 12.75 & 16.04 & 16.76 & 15.31 & 18.67 & 19.39 & 17.90 & 21.32 \\
\hline & & 20 & 15.57 & 14.28 & 17.62 & 18.29 & 16.98 & 20.27 & 20.93 & 19.57 & 22.89 \\
\hline & & 25 & 16.96 & 15.69 & 19.14 & 19.79 & 18.53 & 21.89 & 22.52 & 21.22 & 24.55 \\
\hline & & 30 & 18.23 & 17.01 & 20.53 & 21.16 & 19.96 & 23.39 & 23.99 & 22.77 & 26.09 \\
\hline
\end{tabular}

\section{Example}

From Wu et al. [4], data consisting of the duration of remission achieved by four drugs for treating leukemia are used to illustrate our proposed simultaneous confidence intervals in Theorem 1. The data are listed in Table 2. We regard drugs 1,2 as the treatment groups $\pi_{1}, \pi_{2}$ and drugs 3,4 as the control groups $\pi_{1}^{*}, \pi_{2}^{*}$. 
Table 2. Data for the duration of remission by four drugs.

\begin{tabular}{cccc}
\hline Drug $1\left(\mu_{1}\right)$ & Drug $2\left(\mu_{2}\right)$ & Drug $3\left(\mu_{1}^{*}\right)$ & Drug $4\left(\mu_{2}^{*}\right)$ \\
\hline 1.034 & 2.214 & 4.158 & 5.115 \\
2.344 & 4.976 & 4.025 & 4.498 \\
1.266 & 8.154 & 5.170 & 4.617 \\
1.563 & 2.686 & 11.909 & 4.651 \\
1.169 & 2.271 & 4.912 & 4.533 \\
4.118 & 3.139 & 4.629 & 4.513 \\
1.013 & 2.214 & 3.955 & 7.641 \\
1.509 & 4.480 & 6.735 & 5.971 \\
1.109 & 8.847 & 3.140 & 12.130 \\
1.965 & 2.239 & 12.446 & 4.699 \\
5.136 & 3.473 & 8.777 & 4.914 \\
1.533 & 2.761 & 6.321 & 17.169 \\
1.716 & 2.833 & 3.256 & 5.497 \\
2.778 & 2.381 & 8.250 & 11.332 \\
2.546 & 3.548 & 3.759 & 18.922 \\
2.626 & 2.414 & 5.205 & 13.712 \\
3.413 & 2.832 & 3.071 & 6.309 \\
1.929 & 5.551 & 3.147 & 10.086 \\
2.061 & 3.376 & 9.773 & 9.293 \\
2.951 & 2.968 & 10.218 & 11.787 \\
\hline
\end{tabular}

The longer mean duration of remission time $\left(\mu_{i}\right.$ or $\left.\mu_{j}^{*}, i=1,2, j=1,2\right)$ is desired for this example. Lawless [12] conducted the likelihood ratio asymptotic $\chi^{2}$ test to show that there is a significant difference among the four scale parameters. Thus, the data analysis one-stage multiple comparison procedures with several controls $\mu_{i}-\mu_{j}^{*}, i=1,2, j=1,2$ in Theorem 1 for exponential mean lifetimes under heteroscedasticity can be applied. The required statistics and critical values of $\widetilde{s}_{U}, \widetilde{s}_{L^{\prime}}$ and $\widetilde{s}_{t}$ for $P=0.90,0.95$, and 0.975 are summarized in Table 3.

Table 3. The required statistics and critical values.

\begin{tabular}{cccccc}
\hline Statistics & Drug $\mathbf{1}$ & Drug $\mathbf{2}$ & Statistics & Drug 3 & Drug $\mathbf{4}$ \\
\hline$Y_{i}$ & 1.013 & 2.214 & $Y_{j}^{*}$ & 3.071 & 4.498 \\
$S_{i}$ & 1.238 & 1.530 & $S_{j}^{*}$ & 3.233 & 4.075 \\
$\widetilde{c}$ & 0.204 & & & & \\
& $Y_{1}-Y_{1}^{*}+$ & $Y_{2}-Y_{1}^{*}+$ & & & \\
& $S_{1}-S_{1}^{*}$ & $S_{2}-S_{1}^{*}$ & & \\
& -4.053 & -2.560 & & \\
& $Y_{1}-Y_{2}^{*}$ & $Y_{2}-Y_{2}^{*}+$ & & \\
$P$ & $S_{1}-S_{2}^{*}$ & $S_{2}-S_{2}^{*}$ & & \\
0.900 & -6.322 & -4.829 & & \\
0.950 & $\widetilde{s}_{U}$ & $\widetilde{S}_{L}$ & $\widetilde{S}_{t}$ & & \\
0.975 & 13.02 & 13.03 & 15.70 & & \\
& 15.75 & 15.75 & 18.35 & & \\
& 18.36 & 18.39 & 20.98 & & \\
\hline
\end{tabular}

Applying parts (a) and (b) of Theorem 1, we can find the upper and lower confidence bounds for $\mu_{i}-\mu_{j}^{*}, i=1,2, j=1,2$ with confidence coefficients $0.90,0.95$, and 0.975 given in Table 4. With respect to the upper confidence bounds, drug 1 is selected in a subset of worse than several controls (drug 3 and 4 ) and drugs 1 and 2 are selected in a subset of worse than the control (drug 3 ) with the probability of correct selection being at least 0.90 , 0.95 , and 0.975 in terms of mean lifetimes since their one-sided confidence intervals do not contain zero. 
Table 4. The upper confidence bounds and lower confidence bounds under $P=0.90,0.95,0.975$.

\begin{tabular}{|c|c|c|c|}
\hline \multirow{2}{*}{ Parameter } & \multicolumn{3}{|c|}{$\left(-\infty, Y_{i}+S_{i}-Y_{j}^{*}-S_{j}^{*}+\tilde{c} \tilde{s}_{U}\right),\left(Y_{i}+S_{i}-Y_{j}^{*}-S_{j}^{*}-\tilde{c}_{L}, \infty\right)$} \\
\hline & $90 \%$ & $95 \%$ & $97.5 \%$ \\
\hline 1. $\mu_{1}-\mu_{1}^{*}$ & $\begin{array}{c}(-\infty,-1.397) \\
(-6.711, \infty)\end{array}$ & $\begin{array}{c}(-\infty,-0.840) \\
(-7.266, \infty)\end{array}$ & $\begin{array}{c}(-\infty,-0.320) \\
(-7.805, \infty)\end{array}$ \\
\hline 2. $\mu_{1}-\mu_{2}^{*}$ & $\begin{array}{c}(-\infty,-3.666) \\
(-8.980, \infty)\end{array}$ & $\begin{array}{c}(-\infty,-3.109) \\
(-9.535, \infty)\end{array}$ & $\begin{array}{c}(-\infty,-2.589) \\
(-10.07, \infty)\end{array}$ \\
\hline 3. $\mu_{2}-\mu_{1}^{*}$ & $\begin{array}{l}(-\infty, 0.096) \\
(-5.218, \infty)\end{array}$ & $\begin{array}{l}(-\infty, 0.653) \\
(-5.773, \infty)\end{array}$ & $\begin{array}{l}(-\infty, 1.173) \\
(-6.312, \infty)\end{array}$ \\
\hline 4. $\mu_{2}-\mu_{2}^{*}$ & $\begin{array}{c}(-\infty,-2.173) \\
(-1.616, \infty)\end{array}$ & $\begin{array}{c}(-\infty,-1.616) \\
(-8.042, \infty)\end{array}$ & $\begin{array}{c}(-\infty,-1.096) \\
(-8.581, \infty)\end{array}$ \\
\hline
\end{tabular}

Applying part (c) of Theorem 1, we can find the two-sided confidence bounds for $\mu_{i}-\mu_{j}^{*}, i=1,2, j=1,2$ with confidence coefficients $0.90,0.95$, and 0.975 listed in Table 5 . For confidence coefficients $0.90,0.95$, and 0.975 , we can conclude that drug 1 is worse than several controls (drugs 3 and 4 ) and drug 2 is worse than drug 4 since both bounds of the two-sided confidence intervals for these two drugs are negative. Furthermore, drug 2 is not significantly different from drug 3 in terms of mean remission times.

Table 5. The two-sided confidence intervals under $P=0.90,0.95,0.975$.

\begin{tabular}{cccc}
\hline \multirow{2}{*}{ Parameter } & \multicolumn{3}{c}{$\left(\boldsymbol{Y}_{i}+S_{i}-\boldsymbol{Y}_{j}^{*}-\boldsymbol{S}_{j}^{*}-\tilde{\boldsymbol{c}} \tilde{\boldsymbol{s}}_{t}, \boldsymbol{Y}_{\boldsymbol{i}}+\boldsymbol{S}_{\boldsymbol{i}}-\boldsymbol{Y}_{j}^{*}-\boldsymbol{S}_{j}^{*}+\tilde{\boldsymbol{c}} \tilde{\boldsymbol{s}}_{t}\right)$} \\
\cline { 2 - 4 } & $\mathbf{9 0 \%}$ & $\mathbf{9 5 \%}$ & $\mathbf{9 7 . 5 \%}$ \\
\hline $1 . \mu_{1}-\mu_{1}^{*}$ & $(-7.256,-0.850)$ & $(-7.800,-0.310)$ & $(-8.333,0.227)$ \\
$2 . \mu_{1}-\mu_{2}^{*}$ & $(-9.525,-3.119)$ & $(-10.07,-2.579)$ & $(-10.60,-2.042)$ \\
$3 . \mu_{2}-\mu_{1}^{*}$ & $(-5.763,0.643)$ & $(-6.303,1.183)$ & $(-6.840,1.720)$ \\
$4 . \mu_{2}-\mu_{2}^{*}$ & $(-8.032,-1.626)$ & $(-8.572,-1.086)$ & $(-9.109,-0.549)$ \\
\hline
\end{tabular}

\section{Conclusions}

From the critical values in Table 1 and the equation of $L_{1}=2 \widetilde{c s}_{t}$, it is found that the length of SCI is getting wider when we compare more populations for fixed $P$ and $m$.

In practical applications, we may have several control populations instead of one control population. Applying Lam's $[9,10]$ technique, we propose a multiple comparison procedure with several controls for exponential mean lifetimes under heteroscedasticity. In addition, the critical values for $(k, l)=(2,2),(2,3),(2,4),(3,2),(3,3),(4,2), m=2(1) 10(5) 30$ and $P^{*}=0.90,0.95$, and 0.975 are provided for the use of this investigation problem in Table 1 . Finally, we use one example to illustrate the practical use of our proposed procedures in Theorem 1 . The multiple comparison procedure with one control for exponential mean lifetimes in $\mathrm{Wu}$ [8] is only a special case of our new procedure in Theorem 1 of this research when $l=1$. Therefore, the results in this paper have more general methods in many fields of applications.

Funding: This research was funded by the Ministry of Science and Technology, Taiwan, MOST 108-2118-M-032-001 and MOST 109-2118-M-032-001-MY2 and the APC was funded by MOST 1092118-M-032-001-MY2.

Institutional Review Board Statement: Not applicable.

Informed Consent Statement: Not applicable.

Data Availability Statement: Data available in a publicly accessible repository. The data presented in this study are openly available in $\mathrm{Wu}$ et al. [4].

Conflicts of Interest: The author declares no conflict of interest. 


\section{Appendix A}

Proof of Lemma 1. Consider the following three cases:

Case 1: For $b \geq a,[X \geq Y-d$ and $Y \leq d]$ implies

$$
a X \geq a(Y-d) \geq b(Y-d) \geq b Y-d \max (a, b) .
$$

Case 2: For $b<a$ and $Y \leq 0,[X \geq-d]$ implies

$$
a X \geq-a d \geq b Y-d \max (a, b) .
$$

Case 3: For $b<a$ and $Y>0,[X \geq Y-d]$ implies

$$
a X \geq a(Y-d) \geq b Y-d \max (a, b) \text {. }
$$

The proof is established.

Proof of Theorem 1. For (a), we have

$$
\begin{aligned}
& \mathrm{P}\left(\mu_{i}-\mu_{j}^{*} \leq Y_{i}+S_{i}-Y_{j}^{*}-S_{j}^{*}+\widetilde{c s}_{U}, i=1, \ldots, k, j=1, \ldots, l\right) \\
&= \mathrm{P}\left(\theta_{i}+\sigma_{i}-\eta_{j}-\xi_{j} \leq Y_{i}+S_{i}-Y_{j}^{*}-S_{j}^{*}+\widetilde{c s}_{U}, i=1, \ldots, k, j=1, \ldots, l\right) \\
&= \mathrm{P}\left(-S_{i}+\sigma_{i}+\theta_{i}-Y_{i} \leq-S_{j}^{*}+\eta_{j}+\xi_{j}-Y_{j}^{*}+\widetilde{c s}_{U}, i=1, \ldots, k, j=1, \ldots, l\right) \\
&= \mathrm{P}\left(\frac{S_{i}}{m} \frac{\sigma_{i}}{S_{i}} \frac{m\left(-S_{i}+\sigma_{i}+\theta_{i}-Y_{i}\right)}{\sigma_{i}} \leq \frac{S_{j}^{*}}{m} \frac{\eta_{j}}{S_{j}^{*}} \frac{m\left(-S_{j}^{*}+\eta_{j}+\xi_{j}-Y_{j}^{*}\right)}{\eta_{j}}+\widetilde{c s}_{U}\right. \\
&=\mathrm{P}\left(\frac{S_{j}^{*}}{m} G_{j}^{*} \geq \frac{S_{i}}{m} G_{i}-\widetilde{c s}_{U}, i=1, \ldots, k, j=1, \ldots, l\right) \\
& \geq \mathrm{E}_{S_{1}, \cdots, S_{k}} \mathrm{P}\left(\frac{S_{j}^{*}}{m} G_{j}^{*} \geq \frac{S_{i}}{m} G_{i}-\max \left(\frac{S_{i}}{m}, \frac{S_{j}^{*}}{m}\right) \widetilde{s}_{U}, i=1, \ldots, k, j=1, \ldots, l\right) \\
& \geq \mathrm{P}\left(G_{j}^{*} \geq-\widetilde{s}_{U}, G_{i} \leq \widetilde{s}_{U}, G_{j}^{*} \geq G_{i}-\widetilde{s}_{U}, i=1, \ldots, k, j=1, \ldots, l\right) \\
&=\mathrm{P}\left(-G_{j}^{*} \leq \widetilde{s}_{U}, G_{i} \leq s_{U}^{*}, G_{i}-G_{k} \leq \widetilde{s}_{U}, i=1, \cdots, k, j=1, \ldots, l\right) \\
&=\mathrm{P}\left(\max \left(-G_{j}^{*}, G_{i}, G_{i}-G_{j}^{*}, i=1, \ldots, k, j=1, \ldots, l\right) \leq \widetilde{s}_{U}\right)=P
\end{aligned}
$$

Satisfying the above equation, the value of $\widetilde{s}_{U}$ is determined as the $100 P$-th percentile of the distribution of $\max \left(-G_{j}^{*}, G_{i}, G_{i}-G_{j}^{*}, i=1, \cdots, k, j=1, \ldots, l\right)$ and the proof is thus established.

For (b), we have

$$
\begin{gathered}
\mathrm{P}\left(\mu_{i}-\mu_{j}^{*} \geq Y_{i}+S_{i}-Y_{j}^{*}-S_{j}^{*}-\widetilde{c s}_{L^{\prime}} i=1, \cdots, k, j=1, \ldots, l\right) \\
\mathrm{P}\left(\theta_{i}+\sigma_{i}-\eta_{j}-\xi_{j} \geq Y_{i}+S_{i}-Y_{j}^{*}-S_{j}^{*}+\widetilde{c s}_{L^{\prime}}, i=1, \ldots, k, j=1, \ldots, l\right) \\
=\mathrm{P}\left(-S_{i}+\sigma_{i}+\theta_{i}-Y_{i} \geq-S_{j}^{*}+\eta_{j}+\xi_{j}-Y_{j}^{*}+\widetilde{c s}_{L^{\prime}} i=1, \ldots, k, j=1, \ldots, l\right) \\
=\mathrm{P}\left(\frac{S_{i}}{m} \frac{\sigma_{i}}{S_{i}} \frac{m\left(-S_{i}+\sigma_{i}+\theta_{i}-Y_{i}\right)}{\sigma_{i}} \geq \frac{S_{j}^{*}}{m} \frac{\eta_{j}}{S_{j}^{*}} \frac{m\left(-S_{j}^{*}+\eta_{j}+\xi_{j}-Y_{j}^{*}\right)}{\eta_{j}}+\widetilde{c s}_{\mathrm{L}}\right. \\
, i=1, \ldots, k, j=1, \ldots, l)
\end{gathered}
$$




$$
\begin{gathered}
=\mathrm{P}\left(\frac{S_{i}}{m} G_{i} \geq \frac{S_{j}^{*}}{m} G_{j}^{*}-\widetilde{c s}_{L}, i=1, \ldots, k, j=1, \ldots, l\right) \\
\geq \mathrm{E}_{S_{1}, \cdots, S_{k}} \mathrm{P}\left(\frac{S_{i}}{m} G_{i} \geq i \frac{S_{j}^{*}}{m} G_{j}^{*}-\max \left(\frac{S_{i}}{m}, \frac{S_{j}^{*}}{m}\right) \widetilde{s}_{L}, i=1, \ldots, k, j=1, \ldots, l\right) \\
\geq \mathrm{P}\left(G_{i} \geq-\widetilde{s}_{L}, G_{j}^{*} \leq \widetilde{s}_{L}, G_{i} \geq G_{j}^{*}-\widetilde{s}_{L}, i=1, \ldots, k, j=1, \ldots, l\right) \\
\geq \mathrm{P}\left(-G_{i} \leq \widetilde{s}_{L}, G_{j}^{*} \leq \widetilde{s}_{L}, G_{j}^{*}-G_{i} \leq \widetilde{s}_{L}, i=1, \ldots, k, j=1, \ldots, l\right) \\
=\mathrm{P}\left(\max \left(-G_{i}, G_{j}^{*}, G_{j}^{*}-G_{i}, i=1, \ldots, k, j=1, \ldots, l\right) \leq \widetilde{s}_{L}\right)=P
\end{gathered}
$$

Satisfying the above equation, the value of $\widetilde{s}_{L}$ is determined as the 100P-th percentile of the distribution of $\max \left(-G_{i}, G_{j}^{*}, G_{j}^{*}-G_{i}, i=1, \cdots, k, j=1, \ldots, l\right)$ and the proof is thus established.

For (c), combining (a) and (b), we have

$$
\begin{gathered}
\mathrm{P}\left(Y_{i}+S_{i}-Y_{j}^{*}-S_{j}^{*}-\widetilde{c s}_{t} \leq \mu_{i}-\mu_{j}^{*} \leq Y_{i}+S_{i}-Y_{j}^{*}-S_{j}^{*}+\widetilde{c s}_{t}, i=1, \cdots, k, j=1, \ldots, l\right) \\
=\mathrm{P}\left(Y_{i}+S_{i}-Y_{j}^{*}-S_{j}^{*}-\widetilde{c s}_{t} \leq \theta_{i}+\sigma_{i}-\eta_{j}-\widetilde{\xi}_{j} \leq Y_{i}+S_{i}-Y_{j}^{*}-S_{j}^{*}+\widetilde{c s}_{t},\right. \\
, i=1, \cdots, k, j=1, \ldots, l) \\
=\mathrm{E}_{S_{1}, \cdots, S_{k}} \mathrm{P}\left(-G_{i} \leq \widetilde{s}_{t}, G_{j}^{*} \leq \widetilde{s}_{t}, G_{j}^{*}-G_{i} \leq \widetilde{s}_{t} \cap\right. \\
\left.-G_{j}^{*} \leq \widetilde{s}_{t}, G_{i} \leq \widetilde{s}_{t}, G_{i}-G_{j}^{*} \leq \widetilde{s}_{t}, i=1, \cdots, k, j=1, \ldots, l\right) \\
\geq \mathrm{P}\left(\max \left(\left|G_{i}\right|,\left|G_{j}^{*}\right|,\left|G_{j}^{*}-G_{i}\right|, i=1, \cdots, k, j=1, \ldots, l\right) \leq \widetilde{s}_{t}\right)=P
\end{gathered}
$$

Satisfying the above equation, the value of $\widetilde{s}_{t}$ is determined as the $100 P$-th percentile of the distribution of $\max \left(\left|G_{i}\right|,\left|G_{j}^{*}\right|,\left|G_{j}^{*}-G_{i}\right|, i=1, \ldots, k, j=1, \ldots, l\right)$ and the proof is thus established.

\section{References}

1. Bechhofer, R.E. A single sample multiple decision procedure for ranking means of normal populations with known variances. Ann. Math. Stat. 1954, 25, 16-39. [CrossRef]

2. Gupta, S.S. On a Decision Rule for a Problem in Ranking Means. Doctoral Dissertation, Mimeograph Series No. 150. Institute of Statistics, University of North Carolina, Chapel Hill, NC, USA, 1956.

3. Lam, K.; Ng, C.K. Two-stage procedures for comparing several exponential populations with a control when the scale parameters are unknown and unequal. Seq. Anal. 1990, 9, 151-164. [CrossRef]

4. Wu, S.F.; Lin, Y.P.; Yu, Y.R. One-stage multiple comparisons with the control for exponential location parameters under heteroscedasticity. Comput. Stat. Data Anal. 2010, 54, 1372-1380. [CrossRef]

5. Wu, S.F. New one-stage multiple comparisons procedures with the average for exponential location parameters under heteroscedasticity. J. Stat. Comput. Simul. 2016, 86, 2740-2748. [CrossRef]

6. Maurya, V.; Goyal, A.; Gill, A.N. Multiple comparisons with more than one control for exponential location parameters under heteroscedasticity. Commun. Stat. Simul. Comput. 2011, 40, 621-644. [CrossRef]

7. Maurya, V.; Gill, A.N.; Singh, P. Multiple comparisons with a control for exponential location parameters under heteroscedasticity. J. Appl. Stat. 2013, 40, 1817-1830. [CrossRef]

8. Wu, S.F. One stage multiple comparisons of k-1 treatment mean lifetimes with the control for exponential distributions under heteroscedasticity. Commun. Stat. Simul. Comput. 2018, 47, 2968-2978. [CrossRef]

9. Lam, K. Subset Selection of Normal Populations under Heteroscedasticity. IPASRAS-II: Proceedings and Discussions of the Second International Conference on Inference Procedures Associated with Statistal Ranking and Selection on the Frontiers of Modern Statistical Inference Procedures, II; ACM: New York, NY, USA, 1992; pp. 307-344.

10. Lam, K. An improved two-stage selection procedure. Commun. Stat. Simul. Comput. 1988, 17, 995-1006. [CrossRef]

11. Gail, M.H.; Gastwirth, J.L. A scale-free goodness-of-fit Test for the Exponential Distribution Based on the Gini Statistic. J. R. Stat. Soc. Ser. B 1978, 40, 350-357. [CrossRef] 
12. Lawless, J.F. Statistical Models and Methods for Lifetime Data; Wiley: New York, NY, USA, 2003.

13. Roussas, G.G. A Course in Mathematical Statistics; Academic Press: San Diego, CA, USA, 1997. 